\title{
The Effect of Kinesio Taping on Pain and Jump Performance in Club Volleyball Players with Patellar Tendinopathy in the Cayman Islands \\ D Martin ${ }^{1}$, G Nelson ${ }^{2}$, A Mansingh $^{1}, \mathrm{~N} \mathrm{Wade}^{1}$
}

\begin{abstract}
Affiliations:
${ }^{1}$ Division of Sports Medicine, Faculty of Medical Sciences, The University of the West Indies, Kingston 7, Jamaica.

${ }^{2}$ School of Physiotherapy, Department of Basic Medical Sciences, The University of the West Indies, Kingston 7, Jamaica.
\end{abstract}

\section{Correspondence:}

Dr A Mansingh

Division of Sports Medicine

Faculty of Medical Sciences

The University of the West Indies

Kingston 7

Jamaica.

Fax: 1-876-977-2289, 1876-977-3470

E-mail: akshai.mansingh@uwimona.edu.jm

Short title: Kinesio Taping and Patella Tendinopathy in Volleyball Players

Synopsis: Kinesio taping has gained widespread popularity in clinical, rehabilitation, orthopaedic settings. It has been used as a treatment and performance enhancement tool. The effectiveness of Kinesio-taping in relieving pain experienced in patellar tendinopathy in volleyball players is evaluated and discussed. 


\section{ABSTRACT}

Objective: To examine the effect of kinesio tape on knee pain and jump performance in club volleyball players with patellar tendinopathy in the Cayman Islands.

Methods: A single group pretest posttest experimental study was conducted. Participants were required to perform three vertical jump tests, first with and then without kinesio tape: test sequence was randomized and counterbalanced. Data were analyzed using the paired t-test. Statistical Package for the Social Sciences (SPSS version 16 for windows) was used to conduct the analyses.

Results: Thirty-three (13 females, 20 males) subjects (mean age $26.9 \pm 5$ years) participated. Kinesio tape significantly decreased mean pain scores in jump performance from $24.7 \pm 17.4$ $\mathrm{mm}$ to $4.24 \pm 6.5 \mathrm{~mm}$ on the $100 \mathrm{~mm}$ Visual Analogue Scale $(\mathrm{p}<0.001)$ and increased jump height from $44.5 \pm 8.2 \mathrm{~cm}$ to $47.7 \pm 7.8 \mathrm{~cm}(\mathrm{p}<0.001)$.

Conclusion: The findings of this study support the clinical use of kinesio taping in decreasing pain and improving jump performance in volleyball players with patellar tendinopathy.

Keywords: Kinesio tape, patellar tendinopathy, vertical jump test 


\section{INTRODUCTION}

Patellar tendinopathy was first referred to as 'Jumper's knee' due to its frequency in jumping sports (1). However, this condition can also be found in varying sports which does not involve jumping or change in directions (2). Patellar tendinopathy interferes with sport performance and may prematurely end the athlete's sporting career (3).

The patellar tendon extends from the inferior pole of the patella, as an extension of the tendon of the quadriceps femoris muscle, to the tibial tuberosity(4). Overuse of the patellar tendon can lead to patellar tendinopathy, which is believed by many to be a degenerative condition, the result of excessive load bearing and tensile strain (5). Pain and disuse associated with patellar tendinopathy may lead to poor vastus medialis obliquus (VMO) function and altered patellofemoral joint biomechanics (6). This pain may be further aggravated by activities such as excessive jumping, hopping and bounding. Athletic taping of different varieties is frequently used during the rehabilitation period to improve proprioception, support the knee joint and surrounding soft tissue, and to reduce pain and swelling (2).

Kinesio tape is an elastic adhesive tape devised by Dr. Kenzo Kase and has been used in Asia for over five decades(7). The developers of kinesio tape have claimed that it is different from other traditional elastic tapes, as it allows for improved ventilation and water resistance, with more elasticity and minimal skin discomfort relative to their counterparts. They further claim that the effects of the kinesio tape application may be felt immediately or within the first 24 hours of application (7). The kinesio tape is purported to: provide a positional stimulus through the skin; align fascial tissues; create more space by lifting fascia and soft tissues above an area of pain/inflammation; provide sensory stimulation to assist or limit motion; and assist in the removal of edema by improving lymphatic drainage (7). 
Several interventions have been applied to athletes to improve jump performance, but these have not offered prolonged treatment effects (8). The use of kinesio tape has become popular among athletes and it is proposed that when applied it causes proprioceptive stimulation which can enhance joint range of motion and muscle function during exercise (8). No study has been identified which examined the effect of kinesio taping on jump performance in athletes with patellar tendinopathy. Notwithstanding, it has been observed that kinesio taping has become increasingly popular in its management. The purpose of this study, therefore, was to determine whether kinesio taping improved jump performance in persons diagnosed with patellar tendinopathy. It was hypothesized that there would be a difference in pain levels and jump height after the application of kinesio tape.

\section{SUBJECTS AND METHODS}

A single group pretest posttest experimental study design was conducted. After obtaining ethical approval from The University of the West Indies ethics committee, participants were recruited from volleyball clubs in the Cayman Islands. To be included in the study, participants had to have a diagnosis of patellar tendinopathy from a physician, give a positive finding of patellar tendinopathy during a clinical examination (palpation of patellar tendon) and present with a history of pain arising from the patellar tendon for at least 6 weeks. Participants were excluded if they had back pain during the past six weeks, a history of other musculoskeletal pathology involving the hip, knee or ankle which was currently causing pain and/or disability. All volleyball players gave signed informed consent prior to participating. 
All subjects were required to perform the jump tests with and without kinesio tape on two separate occasions, twenty-four hours apart. The order in which the tests were performed was randomized and counterbalanced. Subjects were tested at the same time on each day. Prior to testing, each subject warmed up for 5 minutes by walking on treadmill at $1.5 \mathrm{~km} / \mathrm{h}$ with no incline. They then conducted three (3) vertical jump tests. The vertical jump test measured the highest distance jumped from a semi-crouched position in the following protocol:

1. The standing reach height was established by asking the subject to stand with the preferred shoulder adjacent to the wall and feet flat on the ground, and reach as high as possible to touch the wall with the middle finger. The subject's fingertips were chalked so that a mark was left on the wall. This starting point represented the distance from this wall mark to the floor, recorded in centimeters $(\mathrm{cm})$.

2. The subject was then instructed to bend the knees to about a $90^{\circ}$ angle, while moving the arms back in a winged position;

3. The subject then jumped upward, touching as high as possible on the wall.

The highest of three jumps performed as outlined above was used as the vertical height attained. The vertical jump height was computed as the difference between the standing reach height and the vertical height achieved in the jump.

At the same time the following day, those subjects who performed the vertical jump test 'with tape' first then did it without and those who performed the test 'without tape' then did it with the tape applied. Those who performed the test with the 'tape first' were sent home with the tape in place for a period of 24 hours and the subject was instructed to return to the clinic the following day at the same time and performed the jump tests. Subjects were instructed to remove the tape prior to the prescribed time only if any persistent skin irritation or increased knee 
discomfort occurred. The researcher supervised the testing of each subject but all testing was done by an independent therapist. The kinesio tape was applied by the researcher (DM). In performing the jump with the kinesio tape, the tape was applied to the affected quadriceps femoris muscle from origin to insertion in a ' $\mathrm{Y}$ strip' application and a ' $\mathrm{U}$ strip' application (Figure 2) as suggested by the developers ${ }^{7}$ for patellar tendinopathy. The average pain score was recorded for each test using a $100 \mathrm{~mm}$ Visual Analogue Scale (VAS).

The paired t-test was used to determine if the mean differences in height (in centimeters) and VAS scores (in millimeters) were significant between each testing scenario. Testing was done at $\alpha$ level of 0.05 .

\section{RESULTS}

Thirty-three (13 females and 20 males) volleyball players participated. The mean age was $26.9 \pm$ 5.2 years. There was an increase in the mean jump height from $44.5 \pm 8.2 \mathrm{~cm}$ without kinesio tape to $47.7 \pm 7.8 \mathrm{~cm}$ with kinesio tape $(\mathrm{p}<0.001)$. This positive change in scores is indicative of improvement in performing the vertical jump test. There was a decrease in the mean pain scores from $24.7 \pm 17.4 \mathrm{~mm}$ without kinesio tape to $4.24 \pm 6.51 \mathrm{~mm}$ with kinesio tape $(\mathrm{p}<0.001)$.

\section{DISCUSSION}

The study sought to determine the effectiveness of kinesio taping in reducing knee pain and improving jump performance in volleyball players diagnosed with patellar tendinopathy. 
Kinesio taping has been used as a treatment and performance enhancement tool. It has gained popularity in clinical, rehabilitation, orthopaedic settings especially on athletes due its beneficial effects of improving joint range of motion, improving performance, reducing pain, and increasing quadriceps femoris strength $(2,8-12)$. On the contrary, although various studies have highlighted the benefits of kinesio taping, there have also been inconsistencies in reports on the effects of Kinesio taping on muscle activity. Fu et al conducted a pilot study that looked at effects of Kinesio taping on muscle strength in athlete where it was postulated that there was no influence on muscle activity in young athletes when Kinesio taping was applied(13). Nunes and colleagues also found that Kinesio taping technique had no effect in improving jumping performance (14). The results of the study demonstrated that kinesio taping was more effective than 'no taping' in reducing knee pain and improving jump performance.

The significant reduction in pain scores observed with the kinesio tape supports other studies which examined the effect of kinesio tape on pain (15-19). A turkey study of 31 women with patellar femoral pain syndrome who were randomized into therapeutic and control group, the study concluded that there was a reduction of pain in both groups (15). A 2009 study conducted by Gonzalez-Iglesiaz et al concluded that kinesi taping significantly decreased pain in patients with acute whiplash (18). Paoloni et al., study in 2011applied Kinesio tape to patients with chronic low back pain and results demonstrated improvement in pain symptoms after application (19). An unpublished study on 51 Bahamian athletes randomized into three groups: rigid taping, kinesio taping and control group performed a step down test before and after taping and pain level documented concluded that rigid taping reduced pain in patients with patellofemoral pain syndrome, but kinesio taping improved performance in functional sportrelated activities (20). Thelen et al.(17) found that kinesio taping had an immediate reduction in 
pain-free shoulder abduction range-of-motion in subjects with rotator cuff tendonitis; however, there was no meaningful reduction in pain. Although Kinesio taping has been reported to be effective in treating sport injuries, it has also been documented to contribute in the management of various conditions such as low back pain, meralgia paresthetica and myofascial shoulder pain (21-23).

Huang et al., examined the effect of the Kinesio tape to muscle activity and vertical jump performance in healthy inactive people and found that although the application of Kinesio taping seemed to increase muscle activation of the medial gastrocnemius muscle it had no significant difference in jump height (9). In this study however, there was a significant increase in jump performance with kinesio tape. Slupik et, al., suggested that after 24 hours of Kinesio taping, the muscle motion unit increases(24), which implies that duration potentially plays a role in increasing muscle strength. Timing and coordination are also other factors to consider in achieving full vertical jump height (9). The application of Kinesio tape increased vertical jump height according to MacDowall et al in 2015 but kinesio taping had no effect on electromyography activities of the gastrocnemius and soleus muscle (25).

The subjects in the study were healthy individuals and the kinesio tape was applied to the calf muscles. There was a significant increase in the muscle activity on electromyography with subjects wearing kinesio tape.

The improvement in jump performance with kinesio tape seen in this current study may have been mediated by pain reduction during the jump; however, the study design does not allow causation to be determined. The experience of pain is subjective and the marked improvement in pain scores could have been exaggerated. Future studies should use a randomized controlled trial to more precisely determine the effect of kinesio tape on jump performance in this population. 


\section{CONCLUSION}

The findings of this study support the clinical use of kinesio taping in decreasing pain and improving jump performance in volleyball players with patellar tendinopathy. 


\section{REFERENCES}

1. Blazina ME, Kerlan RK, Jobe FW, Carter VS, Carlson GJ. Jumper's knee. Orthop Clin North Am 1973;4(3):665-78.

2. Brukner P, Khan K. Clinical Sports Medicine. 3rd ed. Sydney, Australia: McGraw- Hill; 2006.

3. Peers KH, Lysens RJ. Patellar tendinopathy in athletes: current diagnostic and therapeutic recommendations. Sports Med 2005;35(1):71-87.

4. Soldado F, Reina F, Yuguero M, Rodriguez-Baeza A. Clinical anatomy of the arterial supply of the human patellar ligament. Surg Radiol Anat 2002;24(3-4):177-82.

5. Rees JD, Lichtwark GA, Wolman RL, Wilson AM. The mechanism for efficacy of eccentric loading in Achilles tendon injury; an in vivo study in humans. Rheumatology(Oxford) 2008;47(10):1493-7.

6. Khan KM, Forster BB, Robinson J, Cheong Y, Louis L, Maclean L, et al. Are ultrasound and magnetic resonance imaging of value in assessment of Achilles tendon disorders? A two year prospective study. Br J Sports Med 2003;37(2):149-53.

7. Kase K WJ, Kase T. Clinical therapeutic applications of the kinesio taping method. Tokyo, Japan: Ken IKai Co. Ltd; 2003.

8. Murray H, Husk LJ. Effect of kinesio taping on proprioception in the ankle. J Orthop Sports Phys Ther 2001;31(A37).

9. Huang CY, Hsieh TH, Lu SC, Su FC. Effect of the Kinesio tape to muscle activity and vertical jump performance in healthy inactive people. Biomed Eng Online 2011;10:70.

10. Christou EA. Patellar taping increases vastus medialis oblique activity in the presence of patellofemoral pain. J Electromyogr Kinesiol 2004;14(4):495-504. 
11. Lewis JS, Wright C, Green A. Subacromial impingement syndrome: the effect of changing posture on shoulder range of movement. J Orthop Sports Phys Ther 2005;35(2):72-87.

12. Yoshida A, Kahanov L. The effect of kinesio taping on lower trunk range of motions. Res Sports Med 2007;15(2):103-12.

13. Fu TC, Wong AM, Pei YC, Wu KP, Chou SW, Lin YC. Effect of Kinesio taping on muscle strength in athletes-a pilot study. J Sci Med Sport 2008;11(2):198-201.

14. Nunes GS, de Noronha M, Cunha HS, Ruschel C, Borges NG, Jr. Effect of kinesio taping on jumping and balance in athletes: a crossover randomized controlled trial. J Strength Cond Res 2013;27(11):3183-9.

15. Akbas E, Atay AO, Yuksel I. The effects of additional kinesio taping over exercise in the treatment of patellofemoral pain syndrome. Acta Orthop Traumatol Turc 2011;45(5):33541.

16. Kaya E, Zinnuroglu M, Tugcu I. Kinesio taping compared to physical therapy modalities for the treatment of shoulder impingement syndrome. Clin Rheumatol 2011;30(2):201-7.

17. Thelen MD, Dauber JA, Stoneman PD. The clinical efficacy of kinesio tape for shoulder pain: a randomized, double-blinded, clinical trial. J Orthop Sports Phys Ther 2008;38(7):389-95.

18. González-Iglesias J, Fernandez-de-las-Penas C, Cleland JA, Huijbregts P. Short-term effects of cervical kinesio taping on pain and cervical range of motion inpatients with acute whiplash injury: a randomized clinical trial. J Orthop Sports Phys Ther 2009;39:515-21. 
19. Paoloni M, Bernetti A, Fratocchi G, Mangone M, Parrinello L, Del Pilar Cooper M, et al. Kinesio Taping applied to lumbar muscles influences clinical and electromyographic characteristics in chronic low back pain patients. Eur J Phys Rehabil Med. 2011;47:23744

20. Sherwood Wallace K, Mansingh A, Nelson G. Comparison between rigid and kinesio taping in athletes with patellofemoral pain syndrome: A randomised controlled trial. [Master's Thesis]. unpublished.

21. Kalichman L, Vered E, Volchek L Relieving symptoms of meralgia parasthetica using Kinesio taping: A pilot study. Arch Phys Med Rehabil 2010;91:1137-9.

22. Garcia-Muro F, Rodriquez-Fernandez A, Herrero-de-Lucas A. Treatment of myofascial pain in the shoulder with Kinesio taping. A case report. Man Ther 2010;15(3):292-5.

23. Castro-Sanchez AM, Lara--Palomo IC, Mataran-Penarrocha GA, Fernandez-Sanchez M, Sanchez-Labraca N, Arroyo-Morales M. Kinesio taping reduces disability and pain slightly in chronic non-specific low back pain: A randomised trial. J Physiother 2012;58:89-95.

24. Slupik A, Dwornik M, Bialoszewski D, Zych E. Effect of Kinesio Taping on bioelectrical activity of vastus medialis muscle. Preliminary report. Ortop Traumatol Rehabil 2007;9(6):644-51.

25. MacDowall I, Sanzo P, Zerpa C. The Effect of Kinesio Taping on Vertical Jump Height and Muscle Electromyographic Activity of the Gastrocnemius and Soleus in Varsity Athletes. Int J Sports Sci 2015;5(4):162-70. 